\title{
Espacios accesibles en la escuela inclusiva
}

\section{Accessible Spaces in Inclusive Schools}

\author{
María Julieta Solórzano Salas ${ }^{1}$ \\ Sección de Educación Especial \\ Escuela de Orientación y Educación Especial \\ Universidad de Costa Rica \\ San José, Costa Rica \\ julieta.solorzano@ucr.ac.cr
}

Recibido 11 de setiembre de 2012 • Corregido 28 de febrero de 2013 • Aceptado 13 de marzo de 2013

Resumen. Este artículo de revisión bibliográfica trata, específicamente, el tópico de la accesibilidad física, requerimiento indispensable en las instituciones educativas para garantizar entornos inclusivos que permitan, a las personas integrantes de la comunidad educativa, el ejercicio autónomo de sus deberes y derechos. Además, se sintetizan propuestas teóricas -como el diseño universal-, las cuales pueden guiar, en la valoración y consecución de espacios accesibles, al personal de las instituciones educativas y a profesionales vinculados con la gestión del espacio.

Palabras claves. Accesibilidad universal, comunidad escolar, infraestructura, evaluación, Revista Electrónica Educare, Revista Electrónica Educare, Costa Rica.

Abstract. This literature review is focused on physical accessibility, a basic requirement in educational institutions to ensure that all members of the education community have access to inclusive settings, where they can exercise their duties and rights by themselves. In addition, it summarizes theoretical proposals -the universal design, for instance-, which can guide staff members from these institutions and professionals related to space management, in assessing and achieving appropriate spaces.

Keywords. Universal accessibility, school community, infrastructure, evaluation, Educare Electronic Journal, Costa Rica.

1 Máster en Evaluación Educativa, Universidad de Costa Rica. Licenciada en Docencia, Universidad Estatal a Distancia. Bachiller en Ciencias de la Educación con énfasis en Educación Especial, Universidad de Costa Rica. Coordinadora de la carrera de Bachillerato en Educación Especial (Acreditado por SINAES). Coordinadora de la Comisión de Autoevaluación y Mejoramiento, Universidad de Costa Rica. Trabajó en servicios de apoyo educativo del MEP en zonas rurales y urbanas. Actualmente, profesora universitaria de la carrera de Educación Especial, Sede Rodrigo Facio y Recinto de Guápiles. Experiencia como investigadora en actitudes hacia la inclusión educativa, la evaluación de competencias y la comprensión lectora. 
URL: http://www.una.ac.cr/educare

CORREO: educare@una.cr

\section{Introducción}

En el ámbito de la educación inclusiva confluyen una diversidad de factores políticos, actitudinales y curriculares que condicionan y determinan la puesta en práctica de su filosofía educativa. De estos elementos, en este artículo interesa considerar las características de espacios accesibles en la institución educativa donde se desarrollan las experiencias de enseñar y de aprender. Por sí solo, este no constituye un factor determinante en el propósito de alcanzar escuelas inclusivas, pero sí resulta pertinente en un proyecto de esta naturaleza.

Domènech y Viñas $(2007$, p. 8) indican que “(...) el tratamiento de la diversidad, uno de los ejes claves de los nuevos planteamientos, implica la necesidad de una multiplicidad y una pluralidad de funciones y, por lo tanto, una gran flexibilidad en el espacio escolar." Dicha flexibilidad se interpreta, en esta propuesta, como la accesibilidad que garantiza el uso equitativo de los diferentes espacios por parte de las personas que integran la comunidad escolar, es decir, estudiantes, docentes, familias, personal administrativo y otros miembros vinculados. Con frecuencia se escuchan las valoraciones negativas de las condiciones presentes en la infraestructura de los centros educativos nacionales, a pesar de existir presupuesto para su restauración e interés de quienes tienen a cargo mejorar las condiciones.

La UNESCO (2003, p. 11), en relación con los centros escolares y su accesibilidad advierte:

La gran mayoría de los establecimientos de enseñanza son materialmente inaccesibles para muchos alumnos, especialmente los que tienen alguna discapacidad física. En las zonas más pobres, en particular las rurales, muchas veces son inaccesibles, en gran parte debido al mal estado de los edificios o a su escaso mantenimiento, que los vuelve insalubres o poco seguros para todos los alumnos.

Conseguir una efectiva accesibilidad escolar es responsabilidad ineludible del centro escolar y de su organización interna, por ejemplo, la junta de padres y madres. Esta categoría no solo está orientada a facilitar el acceso físico, es decir, a remover las barreras arquitectónicas; también se refiere a las posibilidades de comunicarse con el medio circundante, a las condiciones que facilitan la instalación y uso de la tecnología y del transporte. Juncà (2005, p. 102) plantea que "[La accesibilidad universal] abarca los ámbitos de la edificación, las vías y espacios públicos, parques y jardines, entorno natural, transporte, señalización, comunicación y prestación de servicios". Por tanto, el propósito del presente artículo es reflexionar sobre la importancia de una infraestructura escolar accesible y ofrecer una propuesta para evaluar este entorno con la participación de la comunidad educativa. 


\section{Accesibilidad física}

El concepto de accesibilidad que sirve de guía a la presente propuesta coincide con la definición que ofrecen De Rojas y Santos (2005, p. 295), para quienes la accesibilidad es la "(...) característica que permite el uso y disfrute de un entorno a cualquier persona, con independencia de su condición física, sensorial o intelectual".

Del mismo modo que lo explica Ureña (2007), se parte de una concepción holística en la valoración de los espacios físicos, puesto que, de manera integral, cada persona debe tener acceso a los lugares de la comunidad, aún más a aquellos de los centros educativos en los cuales desarrolla plenamente sus capacidades.

Conviene que, desde su entrada principal, la institución educativa permita a las personas de la comunidad educativa la posibilidad de ejercer su derecho de libre tránsito, en razón de lo cual debe eliminar cualquier barrera que impida garantizar dicho derecho.

Aunado a la visión holística, se considera la institución educativa desde un paradigma ecológico, es decir; el ambiente escolar proporciona riqueza de estímulos que inciden en el aprendizaje, los cuales trascienden los materiales educativos y la lección impartida, tal y como lo comenta Heredero (2008, p. 296):

Concebimos que la escuela es un todo, es un ambiente educativo global, es un organismo vital y por tanto entre sus partes tiene que haber una comunicación ordenada para los receptores de cualquier tipo de mensaje. El espacio en el que se desarrolla el proceso de enseñanza-aprendizaje es más que su mero continente físico, pues los estímulos que transmiten inciden en la calidad misma del proceso.

Uno de los elementos por considerar cuando se habla de accesibilidad en la infraestructura educativa es el relacionado con la calidad. Si todas las personas participan en un mismo entorno, se puede afirmar que se favorece una mayor integración de la comunidad educativa y es posible que se manifieste un mayor aprovechamiento de los espacios al realizar actividades diversas como ferias y deportes. Se supera, así, la imagen de que la escuela funciona únicamente para la reproducción del currículo. Como lo indica Domènech y Viñas (2007, p. 13), calidad es también la "(...) utilización eficaz de los recursos (...)" y agregan que, en muchas comunidades, la escuela es el único espacio que provee las condiciones necesarias para la cultura y el deporte, posición que también comparte Heredero (2008, p. 300): 
URL: http://www.una.ac.cr/educare

CORREO: educare@una.cr

Es necesario que el centro escolar se convierta en centro de la vida sociocultural, poniendo sus recursos a disposición de jóvenes y adultos: bibliotecas, instalaciones deportivas, salones de actos y otros servicios, tanto escolares como comunitarios, son susceptibles de un aprovechamiento mayor $y$, por tanto, de una rentabilidad superior a la que ofrecen sirviendo exclusivamente a tareas docentes. Ha de romperse la barrera formal que todavía existe entre escuela y comunidad, barrera incomprensible que colabora al aislamiento de aquélla de las realidades de su entorno.

Por lo tanto, es necesario que los centros escolares identifiquen los espacios que limitan el desarrollo pleno de las habilidades individuales y colectivas. A su vez, se requiere la realización de propuestas de intervención alcanzables para erradicar las barreras de acceso a aquellas personas que, por una condición asociada de orden sensorial, física o intelectual, no pueden acceder cómodamente a los diferentes ambientes de la infraestructura escolar.

\section{Legislación nacional en materia de accesibilidad física}

En Costa Rica existe un creciente interés por el tema de la accesibilidad en los centros escolares. Se percibe como un eje transversal presente en diferentes normativas y regulaciones establecidas.

En el Reglamento de Construcciones del INVU se establecen los lineamientos mínimos que requiere el diseño de la planta física. A su vez, esto se regula por el criterio técnico del Ministerio de Educación Pública (MEP) y del Ministerio de Obras Públicas y Transportes (MOPT) (Alfaro, 2003). Este documento indica, en su artículo XI.I, que las instalaciones escolares deben "(...) estar protegidas de los elementos perturbadores de la tranquilidad y salud de los educandos" (Alfaro, 2003, p. 302)".

Por su parte, la Ley 7600 Igualdad de oportunidades para las personas con discapacidad (Asamblea Legislativa de la Repúblicade Costa Rica, 1996) también insiste en la accesibilidad como requisito para la igualdad de oportunidades, y para garantizar la participación de las personas en los diferentes espacios de interacción y en idénticas circunstancias tales como el transporte, la educación, la salud, el espacio físico, la cultura, el deporte, las actividades recreativas, la información y la comunicación. Resulta evidente la consideración de otros ámbitos que deben garantizar la accesibilidad, los cuales son pertinentes y necesarios; no obstante, para efectos de este artículo, se enfoca, particularmente, el espacio físico. 
Por su parte, la Ley 8661 Convención sobre los derechos de las personas con discapacidad (Asamblea Legislativa de la República de Costa Rica, 2007), de reciente implementación en el ámbito nacional, establece que la accesibilidad a los espacios reconocidos por la Ley 7600 que garantiza el goce pleno de los derechos y la igualdad de condiciones. En el artículo 9, referido únicamente a la accesibilidad, dicha ley señala las responsabilidades que debe asumir el Estado, para identificar y eliminar las barreras al acceso adecuado.

Sin embargo, en el contexto costarricense, aún faltan acciones que mitiguen las necesidades identificadas en la investigación llevada a cabo por la Agencia de Cooperación Internacional del Japón y el Consejo Nacional de Rehabilitación y Educación Especial (CNREE) (2006). De particular interés resulta rescatar, del informe citado, que -dentro de los edificios públicos- las escuelas, los colegios y las universidades se ubican en un tercer lugar de las instituciones visitadas por personas con alguna discapacidad. Estos lugares son el destino de un $46 \%$ y de un $40 \%$ de las personas que participaron en la investigación, de las cuales un 37,7\% indica dificultades en el desplazamiento en los centros educativos y un $37.1 \%$ en edificios públicos.

Ante este panorama, que constituye una alerta, es necesario retomar el tema de la accesibilidad en las instituciones educativas, en vista de que urgen los cambios de actitud que propicien la conciencia social y la igualdad de oportunidades para que todas las personas que asisten a la institución educativa se sientan bienvenidas y acogidas, y no enfrenten dificultades en el desplazamiento.

\section{La accesibilidad universal en la institución escolar}

Juncà (2005) explica que el padre del concepto de accesibilidad universal es Ronald L. Mace, quien en 1985 propuso los principios del término. En relación con este, Juncà (2005, p. 102) explica que:

(...) consiste en planear, proyectar, construir, rehabilitar y conservar el entorno de modo que tenga en cuenta la envolvente de necesidades y requerimientos de cualquier persona sea cual sea su edad, circunstancia o capacidades.

Un entorno acorde con los principios de la Accesibilidad Universal (...) será un entorno que facilite el desenvolvimiento y uso del mismo por cualquier persona, desde unas características de comodidad, seguridad y autonomía personal.

Por su parte, Guillamón (2003) plantea que al plantearse el principio de accesibilidad institucional debe hacerse desde el marco de la universalidad. La accesibilidad universal no solo busca dar respuestas a aquellos sectores de la población que presentan una condición 
URL: http://www.una.ac.cr/educare

CORREO: educare@una.cr

de discapacidad física, sensorial o intelectual asociada, sino que contempla, a su vez, otros grupos sociales, como los de la persona adulta mayor, la persona con sobrepeso y las mujeres embarazadas. Al estimar estos otros grupos de la población se puede comprender de una mejor manera la necesidad de crear ambientes accesibles, puesto que cualquiera puede presentar las condiciones indicadas.

De Asís (2008, p. 61) explica que la “(...) accesibilidad ha dejado de ser abordada desde la toma de medidas especiales dentro de un proceso de rehabilitación individual de un sujeto, pasando a serlo desde la búsqueda de respuestas adecuadas, en condiciones de igualdad, a las necesidades de todas las personas".

A partir de los aportes teóricos señalados, es deseable que las sociedades se diseñen de modo que permitan la participación activa de la población que en ella se desarrolla. La escuela idealmente es el modelo de esta premisa fundamentada en la equiparación de oportunidades, y en la cual se reproducen y se multiplican patrones sociales; de ahí que sea tan importante iniciar con la transformación de estos espacios para impactar en la sensibilidad de las nuevas generaciones y en las demás personas que la visitan.

A los centros educativos les corresponde gestar acciones que permitan evaluar las posibilidades de accesibilidad ofrecidas a la comunidad que participa en la escuela -entiéndase profesorado, estudiantado, familiares y otros-. Luego de esa evaluación comprometida -y preferiblemente consensuada por los miembros de la comunidad-, es necesario proponer el listado de actividades que perfilarán una escuela lo más accesible, según los recursos con los que se disponga.

Para la evaluación de las necesidades del centro escolar pueden resultar orientadores los ocho principios del diseño universal. Según Víquez (s. f), estos principios, fundamentales e ineludibles, son: el uso equitativo; la flexibilidad en el uso; la información perceptible; el tamaño y el espacio para la aproximación y uso; el uso simple e intuitivo: ser altamente seguras; implicar bajo esfuerzo físico, especialmente para quienes requieren de aditamentos tales como silla de ruedas o el apoyo del bastón, y poseer dimensiones apropiadas. Para este último, se recomienda que cada institución consulte los lineamientos nacionales sobre las especificaciones acordadas por el Colegio Federado de Ingenieros y Arquitectos.

Del mismo modo Guillamón (2003) propone la calidad del diseño, la funcionalidad, la seguridad, la vida independiente, la comodidad y la innovación como rasgos de un diseño universal.

Además incluye una condición más: la normalización, estrechamente relacionada con el cuarto punto, es decir, con vida independiente; porque en el tanto una persona ejerza de manera autónoma sus deberes y derechos, se puede asegurar que lleva un proceso de vida normalizado.

Tanto los principios como los rasgos expuestos definen una serie de condicionantes que, en caso de presentarse, pueden potencializar la participación de quienes se acercan a la institución escolar. Los cambios por realizar dependen de la posibilidad económica y del recurso humano de la institución educativa. 
Es necesario considerar que algunas de las edificaciones que requieren modificaciones importantes para que alcancen un funcionamiento idóneo, desde el punto de vista de la accesibilidad, y que son consideradas por algún valor arquitectónico particular debido a su historia o estética, requieren de una valoración por parte de las autoridades gubernamentales y de las personas profesionales correspondientes del diseño, para concebir la remodelación que impacte de manera positiva el inmueble.

\section{Consideraciones iniciales para comprender la accesibilidad aplicada a los espacios escolares}

Para la implementación de acciones que garanticen la accesibilidad, resulta pertinente revisar algunos principios vinculados con el tema.

Según García (2005), las actividades que realizan las personas tienen dos componentes, estos se aplican a los contextos escolares:

- El desplazamiento: es el traslado hacia el destino u objeto de interés.

- El uso que se le da a cada aposento en el centro escolar, es decir, las acciones que se ejecutan en determinado espacio.

Es deseable que el desplazamiento y el uso de las instalaciones permitan el máximo aprovechamiento de lo que el medio ofrece. Evidentemente, las barreras arquitectónicas presentes en el diseño de itinerarios limitan el logro de ambos componentes. Para quienes realizan evaluaciones sobre accesibilidad institucional, es importante considerar las necesidades actuales y futuras que presentan los usuarios en el centro escolar.

García (2005) explica que puede resultar complejo proyectar las futuras necesidades en el espacio físico, debido a la diversidad de actividades que se pueden desarrollar y de personas que participan. Recomienda utilizar como criterio los siguientes indicadores funcionales que pueden facilitar la evaluación de las zonas escolares: que una actividad pueda ser realizada por personas sentadas, sin necesidad de movilizarse constantemente, prescindiendo de habilidades motoras finas como al usar los grifos e interruptores y, finalmente, que dichas actividades se puedan realizar sin un sentido básico como la vista o el oído

Por su parte, la Oficina para la Integración de Personas con Discapacidad de la Universidad Complutense de Madrid (s. f) resume las limitaciones más frecuentes que pueden afectar a una persona, sean temporales o permanentes, así: 
URL: http://www.una.ac.cr/educare

CORREO: educare@una.cr

- Maniobra: se relaciona con la posibilidad de ingresar a diferentes espacios y de contar con una adecuada movilidad.

- Desniveles: se refiere a la capacidad de las personas para hacer un recorrido en espacios en donde hay diferentes niveles u obstáculos.

- Alcance: considera la facilidad con que cuenta la persona para alcanzar los objetos que requiere.

- Control: toma en consideración la capacidad de las personas para realizar movimientos precisos, por ejemplo el uso de sus manos.

- Percepción: relacionado con las habilidades sensoriales que le permiten a la persona movilizarse de manera independiente.

Al proponer una evaluación del edificio escolar, lo anterior se constituye en una guía para el profesorado. Igualmente, muestra que para definir las soluciones y establecer la toma de decisiones, resulta indispensable recurrir al criterio del especialista en el campo del diseño espacial.

\section{Interdisciplinariedad en el logro de la accesibilidad universal}

Tradicionalmente el tema de la accesibilidad ha recaído en personas expertas del diseño del espacio, como los profesionales en arquitectura. Al respecto propone Víquez (s. f.):

Con el fin de garantizar la accesibilidad al entorno, los diseñadores y responsables de obras deben concebir, planear y construir los itinerarios para el usuario, tomando en cuenta las diferentes condiciones biológicas, sociales, culturales y antropométricas de todas las personas. (p. 1)

Es decir, los especialistas del diseño de espacios educativos requieren conocer y comprender las necesidades de las personas usuarias de dichos entornos: exigen una visión amplia de la diversidad de demandas del estudiantado, docentes u otros visitantes.

En la consecución de un diseño de mayor calidad, concebido con espacios accesibles y sin barreras, las personas que van a hacer uso de las instalaciones pueden aportar a partir de sus experiencias. Según Domènech y Viñas (2007, p. 8), una de las quejas constantes del profesorado es "(...) la opinión de que los centros no han sido pensados realmente para la función que deben cumplir (...)". 
La cruda realidad muestra que la mayoría de las edificaciones educativas presenta diversas barreras de accesibilidad: alcantarillas sin tapas; obstáculos en los pasos peatonales como bancas, pilas, barandas, y rampas con inclinaciones mayores al $10 \%$ de pendiente proporcional a la longitud. Por lo tanto, se requiere que la comunidad educativa incluya, en su proyecto institucional, las acciones que involucren a la comunidad escolar en la evaluación de sus espacios, de modo que esta tarea sea asumida con el compromiso y el entusiasmo requerido. En este sentido, Heredero (2008, p. 50) plantea:

Es frecuente encontrarnos con edificios de reciente factura en los que sistemáticamente se repiten ciertos errores. El profesorado y alumnado que los habita se van a encontrar a los pocos días o meses con nuevas obras de adaptación y todos los problemas añadidos. Está claro que es imposible en la mayoría de los casos rectificar los proyectos originales y en otros contar con la opinión del profesorado que los habitará pero al menos podían ser analizados los diseños por grupos de profesores que diesen su opinión no desde el punto de vista técnico sino funcional.

Por su parte, Guillamón (2003) y el Consejo de la Tierra (Proyecto de accesibilidad en áreas protegidas para personas con discapacidad) (2002) exponen que se requiere de un enfoque interdisciplinario para lograr una visión integral del concepto de accesibilidad. Es relevante, entonces, la participación de profesionales de diferentes áreas como la medicina, la rehabilitación o la educación.

Para cerrar este apartado, se retoman las palabras de Molina $(2008$, p. 34$)$ sobre otros puntos globales por considerar para lograr una propuesta real de accesibilidad universal:

El diseño para todos es una filosofía e implica un cambio de mentalidad tanto en el diseñador como en el consumidor, por lo que el camino de su implementación abarcará varios ámbitos, como por ejemplo la Enseñanza (modificación de currículo académico), la sociedad civil (difusión, concienciación y conocimiento de los derechos) y la Administración (formación, concienciación, normalización). Una de las posibles estrategias para lograr esto es trabajando en el cambio de mentalidad de los ciudadanos.

Molina (2008) invita a valorar la importancia de la accesibilidad desde diferentes aristas, las cuales permiten afirmar que garantizarla afianza, a su vez, el respeto por los Derechos Humanos de las personas: el tránsito libre y oportuno a un espacio -sin importar las condiciones físicas o sensoriales que pueda presentar la persona- favorece el desarrollo de una vida digna y satisfactoria; es decir, impacta en la calidad de vida. De este modo, al ser el centro educativo 
URL: http://www.una.ac.cr/educare

CORREO: educare@una.cr

uno de los espacios que favorecen la obtención de los conocimientos formales establecidos en un proyecto educativo nacional y otros propios del currículo oculto, surge la necesidad de plantear, a nivel social, el compromiso por brindar entornos adecuados -en los cuales se superen las barreras arquitectónicas del edificio escolar- que permitan el acceso al aprendizaje.

De Asís (2008) expone que las barreras arquitectónicas, las cuales inciden directamente en la accesibilidad, violentan la posibilidad de la persona en el goce de su independencia; en este sentido, se puede anotar que "(...) la falta de accesibilidad o de ajustes razonables implica la negación u obstaculización del reconocimiento, disfrute o ejercicio de los derechos económicos, sociales o culturales, y por ende es un factor en la creación de situaciones de dependencia" (p. 59).

Ahora bien, es necesario reconocer los esfuerzos que los países han realizado en materia de accesibilidad en las instalaciones, especialmente en aquellas que ofrecen servicios públicos. Pero, sin duda, aún queda mucho por hacer en este campo y falta compromiso en la adopción de políticas.

\section{Aspectos mínimos requeridos para la accesibilidad en el centro escolar}

De acuerdo con las ideas desarrolladas hasta este punto y con el fin de concretar una guía para la reflexión institucional que genere una escuela con accesibilidad escolar, la autora del presente artículo propone tres grandes ejes básicos para la evaluación del espacio.

Cada uno de estos ejes promueve acciones específicas que, a su vez, se encuentran estrechamente vinculadas, pues cada uno garantiza la presencia del otro. Por ejemplo, si las instalaciones no son seguras, no se puede garantizar un acceso equitativo, ya que las personas pueden correr riesgos en ciertas áreas. En el caso de un evento sísmico, si la señalización solo informa a algunos usuarios, el resto tendría dificultades para ubicar las puertas de evacuación, lo cual pondría en riesgo a quienes no tienen acceso a la información.

A continuación se explica cada uno de los ejes propuestos:

1. Acceso equitativo a todos los espacios institucionales. Como se ha propuesto anteriormente, es deseable que la escuela sea accesible para todas y todos, es decir, los diferentes espacios físicos institucionales deben permitir la participación de la comunidad escolar. Por lo tanto, la reflexión puede ayudar a identificar la presencia de barreras para ingresar a las aulas, acceder a los patios, a la biblioteca, a los lavados, a la dirección y a la sala de profesores. Es necesario considerar, en todo momento, las posibles acciones que garantizan la accesibilidad: rampas, letreros, barandas, recursos tecnológicos como elevadores, entre otras. 
2. Seguridad en las instalaciones educativas. Se refiere a aquellos espacios que pueden presentar riesgo de accidentes. Tal es el caso de superficies deslizantes, rampas con pendientes elevadas, elementos de tendido eléctrico, postes ubicados en vías peatonales, cambios de nivel poco perceptibles, ausencia de barandas de apoyo en gradas, etcétera.

3. Señalización perceptible. Es necesario que los diferentes espacios estén claramente identificados. Se recomienda recurrir a elementos visuales, auditivos y táctiles que, según sus habilidades, permitan a las personas reconocer los diferentes espacios escolares y las zonas de seguridad. Al respecto, Guillamón (2003, p. 8) recomienda aplicar"(...) contrastes eficaces de color, grafismos de fácil legibilidad y procurando sistemas alternativos que faciliten la accesibilidad a personas con discapacidad visual, auditiva o intelectual".

Existen tablas con símbolos universales que pueden favorecer la señalización escolar. Se deben hacer esfuerzos para facilitar apoyos para las deficiencias sensoriales, como por ejemplo el uso de timbres para las personas con ceguera o baja visión y de luces para las personas sordas.

En fin, las instituciones educativas deben permitir que sus instalaciones sean simplemente accesibles, respondiendo de algún modo a lo que Casado (2003) Ilama "el diseño personalizado", entendido como las acciones y dispositivos que responden a las necesidades de un grupo diverso de personas, que interáctua en un centro educativo.

\section{El diagnóstico de las necesidades en el contexto escolar}

A continuación se propone una guía para facilitar el diagnóstico de las necesidades en el área de la accesibilidad en el contexto escolar. Esta integra los ejes de reflexión señalados.

Se recomienda formar dos o tres subgrupos integrados por diferentes representantes de la comunidad escolar: estudiantado, profesorado, familias, representantes municipales y un profesional en arquitectura. En la propuesta realizada por Consejo de la Tierra (Proyecto de accesibilidad en áreas protegidas para personas con discapacidad) (2002) se favorece la presencia de personas con diferentes edades y géneros.

Respecto a la participación de estudiantes en este tipo de ejercicios Domènech y Viñas (2007, p. 28) señalan:

El alumnado debe participar en la adecuación de los espacios de la escuela. Si la organización del mobiliario está en función de sus necesidades educativas, estos [sic] deben poder apropiarse del espacio, a partir de la posibilidad de cambiar la distribución de los elementos que los complementan. 
URL: http://www.una.ac.cr/educare

CORREO: educare@una.cr

Una vez organizados los grupos de trabajo, cada uno realiza un recorrido por la institución y visita los espacios que la componen. Se procede a enlistar y analizar los obstáculos a la accesibilidad que se presentan, considerando en primer lugar a los diferentes usuarios y sus características específicas (por ejemplo, la edad, la ocupación, la condición física y las discapacidades asociadas, etc.), así como los recursos y posibilidades con que cuenta la institución educativa. Se recomienda elaborar una hoja de registro como la que se presenta a continuación:

Formulario para el diagnóstico de la accesibilidad institucional

\begin{tabular}{|c|c|c|c|c|c|c|c|c|}
\hline \multicolumn{9}{|c|}{ Características para valorar } \\
\hline Usuarios & Edades & \multicolumn{2}{|c|}{$\begin{array}{l}\text { Actividades } \\
\text { que realizan }\end{array}$} & \multicolumn{2}{|c|}{$\begin{array}{l}\text { Necesidades } \\
\text { inmediatas }\end{array}$} & \multicolumn{3}{|c|}{$\begin{array}{c}\text { Soluciones (defininir los apoyos que se } \\
\text { requieren) }\end{array}$} \\
\hline \multicolumn{9}{|c|}{ Estudiantes } \\
\hline \multicolumn{9}{|l|}{ Profesores } \\
\hline \multicolumn{9}{|c|}{$\begin{array}{l}\text { Familias que } \\
\text { nos visitan }\end{array}$} \\
\hline & & & & & & & & \\
\hline \multicolumn{9}{|l|}{ Otros... } \\
\hline \multicolumn{4}{|c|}{ Necesidades proyectadas } & \multicolumn{5}{|c|}{ Soluciones } \\
\hline \multicolumn{9}{|c|}{ Ejes de reflexión } \\
\hline \multirow[t]{2}{*}{$\begin{array}{l}\text { Espacios } \\
\text { escolares }\end{array}$} & \multicolumn{4}{|c|}{$\begin{array}{c}\text { Observaciones sobre los siguientes } \\
\text { ejes }\end{array}$} & \multirow{2}{*}{\multicolumn{2}{|c|}{ Acciones a tomar }} & Estado & Responsables \\
\hline & $\begin{array}{l}\text { Acceso } \\
\text { equitativo }\end{array}$ & Seguro & & ación & & & & \\
\hline \multicolumn{9}{|l|}{ Biblioteca } \\
\hline \multicolumn{9}{|l|}{$\begin{array}{l}\text { Comedor } \\
\text { estudiantil }\end{array}$} \\
\hline Otros... & & & & & & & & \\
\hline
\end{tabular}


Listar las necesidades de los usuarios permitirá visualizar las modificaciones de accesibilidad que requieren los espacios, por lo que, en segundo lugar, se sugiere considerar cada aposento utilizando como guía los ejes planteados.

La autora de este artículo sugiere realizar observaciones descriptivas sobre cada uno de los ejes, lo cual facilitará la definición de las acciones a seguir.

A la hora del análisis de la situación es preciso definir un orden de prioridades para la solución de los problemas detectados. Esto permite calendarizar soluciones y definir responsables para la ejecución y seguimiento de las actividades.

Para finalizar se propone una tabla que puede facilitar la sistematización de los datos recolectados en el análisis y observación de campo, y se sugiere implementar su uso desde la investigación-acción.

\section{Conclusiones}

El centro escolar inclusivo es acogedor y favorece la participación de todas las personas de la comunidad escolar, sin importar las condiciones asociadas a la discapacidad física o sensorial.

Es necesario que los profesionales en el campo de la arquitectura y otras áreas encargadas de la gestión y el diseño del espacio escolar incorporen, en las fases de diagnóstico, el criterio de las personas usuarias: docentes, estudiantes, padres y madres y otras personas de la comunidad escolar.

Interesarse por una infraestructura inclusiva permite garantizar, con un hecho más, el respeto a los derechos humanos de las personas, puesto que la comunidad escolar puede disfrutar de la seguridad y el libre tránsito por las instalaciones educativas.

La propuesta teórica del diseño universal orienta a quienes les corresponde tanto la construcción como la remodelación de los espacios educativos. El propósito consiste en lograr su máximo aprovechamiento; es decir, se pretende trascender de la docencia, y de las relaciones docentes y estudiantes; puesto que interesa una infraestructura en la que toda la comunidad escolar pueda compartir y aprender sin tener que enfrentarse a barreras en la participación.

\section{Agradecimiento}

La autora agradece al arquitecto Alejandro Rodríguez Masís, por la discusión y aportes especializados. 
URL: http://www.una.ac.cr/educare

CORREO: educare@una.cr

\section{Referencias}

Agencia de Cooperación Internacional del Japón (JICA), Consejo Nacional de Rehabilitación y Educación Especial (CNREE). (2006). Las necesidades y oportunidades de las personas con discapacidad en Costa Rica. San José: Autores.

Alfaro, D. (Comp.). (2003). Código urbano (9a ed.) San José, Costa Rica: Editorial Porvenir.

Asamblea Legislativa de la República de Costa Rica. (1996). Ley 7600 Igualdad de oportunidades para las personas con discapacidad. San José: Costa Rica.

Asamblea Legislativa de la República de Costa Rica. (2007). Ley 8661. Convención sobre los derechos de las personas con discapacidad. San José: Costa Rica.

Casado, D. (2003). Accesibilidad con seguridad del medio físico. Ingeniería y territorio, 63, 12-15. Recuperado de http://www.ciccp.es/revistalT/textos/pdf/02_D.Casado_63.pdf

Consejo de la Tierra (Proyecto de accesibilidad en áreas protegidas para personas con discapacidad). (2002). Guía para la realización de un plan de accesibilidaden áreas protegidas. San José, Costa Rica: Fundación Fundecooperación para el desarrollo sostenible.

De Asís, R. (2008). Derechos humanos y situaciones de dependencia. España: Dykinson.

De Rojas, C. y Santos, J. (2005). Mantenimiento y accesibilidad. En Real Patronato sobre Discapacidad y Fundación ACS (Eds.). Manual para un entorno accesible (9a ed., pp. 293310). España: Industrias Gráficas Caro.

Domènech, J. y Viñas, J. (2007). La organización del espacio y del tiempo en el centro educativo $\left(6^{\circ}\right.$ ed.). España: Graó

García, J. (2005). Accesibilidad en la edificación. En Real Patronato sobre Discapacidad y Fundación ACS (Eds.), Manual para un entorno accesible (9a ed., pp.17-35). España: Industrias Gráficas Caro.

Guillamón, J. (2003). La accesibilidad, reto profesional y exigencia social. Ingeniería y Territorio, $63,4-9$.

Heredero, E. S. (2008). A la calidad de la educación desde los espacios escolares: Visión del profesorado y perspectivas en la educación castellano-manchega. España: Universidad de Alcalá.

Juncà, J. (2005). Movilidad accesible. En Real Patronato sobre Discapacidad y Fundación ACS (Eds.), Manual para un entorno accesible (9a ed., pp. 97- 237). España: Industrias Gráficas Caro.

Molina, O. M. (2008). La accesibilidad universal: Un deber de la Universidad de Costa Rica para la inclusión de todas las personas. (Tesis de Maestría). Sistema de Estudios de Posgrado. Universidad de Costa Rica, San José, Costa Rica. 
URL: http://www.una.ac.cr/educare CORREO: educare@una.cr

UNESCO. (2003). Superar la exclusión mediante planteamientos integradores en la educación. Un desafío y una visión. Recuperado de http://www.uam.es/personal_pdi/stmaria/ sarrio/DOCUMENTOS, ARTICULOS, PONENECIAS,/Documento UNESCO sobre educacion inclusiva.pdf

Universidad Complutense de Madrid. (s.f). Accesibilidad y supresión de barreras arquitectónicas. Guía para visualización inmediata. Madrid: Oficina para la Integración de Personas con Discapacidad. Recuperadode http://sid.usal.es/idocs/F8/FDO7637/accesibilidad_ supresion_barreras.pdf

Ureña, L. R. (2007). Accesibilidad universal en las escuelas Buenaventura Corrales y Pilar Jiménez. (Tesis de licenciatura). Escuela de Ingeniería Civil, Facultad de Ingeniería, Universidad de Costa Rica, San José, Costa Rica.

Víquez, M. (s. f). Accesibilidad física. San José, Costa Rica: Consejo Nacional de Rehabilitación y Educación Especial.

\section{Cómo citar este artículo en APA:}

Solórzano, M. J. (2013). Espacios accesibles en la escuela inclusiva. Revista Electrónica Educare, 17(1), 89-103. Recuperado de http://www.revistas.una.ac.cr/index.php/ EDUCARE/issue/current

Nota: Para citar este artículo en otros sistemas puede consultar el hipervínculo "Como citar el artículo" en la barra derecha de nuestro sitio web:

http://www.revistas.una.ac.cr/index.php/EDUCARE/index 